\title{
Multi-service Digital Radio over Fibre System with Millimetre Wave Bridging
}

\author{
Tongyun Li ${ }^{1}$, Haymen Shams ${ }^{2}$, Cyril C. Renaud ${ }^{2}$, Alwyn J. Seeds ${ }^{2}$, Richard Penty ${ }^{1}$, Martyn Fice ${ }^{2}$, Ian White ${ }^{1}$ \\ ${ }^{1}$ Centre for Photonic Systems, Electrical Division, Department of Engineering, University of Cambridge, Cambridge, U.K \\ ${ }^{2}$ Department of Electrical and Electronic Engineering, University College London, London, U.K
}

\begin{abstract}
This paper demonstrates a novel digital radio over fibre (DRoF) architecture that is able to transport multiple compressed digitised RF services using both optical fibre and wireless millimetre wave $(\mathrm{mmW})$ links. This solution has advantages as a cost effective indoor wireless infrastructure where flexible transmission schemes are required. Experimental results indicate wide RF dynamic range for two LTE services transmitted simultaneously, showing its capability for creating a neutral-host radio access network (RAN) with good spectral efficiency and cost effectiveness.
\end{abstract}

Keywords - Millimetre wave, radio-over-fibre, digital signal processing, microwave photonics, high speed wireless.

\section{INTRODUCTION}

Radio over fibre (RoF) technology is a proven solution to deliver multiple wireless carriers over a single network infrastructure for indoor distribution and coverage [1-2]. Digital RoF (DRoF) solutions using common public radio interface (CPRI) have been widely deployed for connecting a baseband unit (BBU) with a remote radio head (RRH) in a cloud radio access network (C-RAN) enabling a centralised and cost-effective digital distribution network architecture. However, the high digital data rates generated in these solutions are causing increasing difficulties for building efficient and converged network infrastructures, especially for $4 \mathrm{G}$ and beyond. It is expected that the CPRI data rate will reach $24.33 \mathrm{Gbps}$ for transporting a 5-sector $4 \times 4$ multiple input multiple output (MIMO) LTE service [3]. Recently, the new eCPRI standard, has been introduced to reduce the data rate by redefining the functional splits between radio equipment control (REC) and radio equipment (RE) [4]. However, this comes at a price of loss of flexibility in operating eCPRI REs (eREs). Another drawback of CPRI is that commercially off-the-shelf CPRI devices are vendor specific. Thus, neutral host or multi-service and multioperator network convergence is not possible currently.

Compressed DRoF techniques for digital distributed antenna system (DDAS) also have benefits for system architecture, as digital signals can be fully regenerated and as a result cascaded links can be used more readily. For example, provided latency requirements are met, links can be cascaded in manners which do not degrade signal quality. Given the growth in high bandwidth mobile services, digital systems can have benefits, for example where the installation of fibre to each remote cell is sometimes difficult for urban cities, mountains, and rivers,

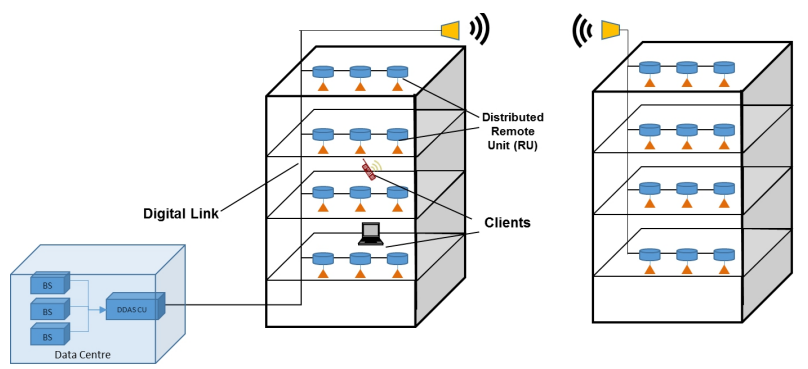

Fig.1. Digital RoF based In-building Wireless System using mmW bridging.

or in a disaster situation where a fast to deploy and reliable connection is needed. Here, wireless millimetre wave $(\mathrm{mmW})$ solutions can be attractive as fronthaul links. However, the communication range is limited by the high free space loss. The integration of $\mathrm{mmW}$ and fibre network can provide a solution for remote cell site coverage in a fronthaul small cell network.

Past research has sought to address this issue, a neutralhost digital RoF system having been previously experimentally demonstrated, using a data compression technique that is able to reduce the digitised LTE signal data rate to $400 \mathrm{Mbps}$ per $20 \mathrm{MHz}$ band LTE channel [5]. The system shows 3-times higher transmission efficiency than CPRI. Based on these digital signal processing algorithms, we have also demonstrated the successful operation of a single service DRoF system over a $40 \mathrm{~km}$ optical fibre link in series with a $1 \mathrm{~m}, 100 \mathrm{GHz} \mathrm{mmW}$ wireless relay. The $\mathrm{mmW}$ link is used as it is envisaged that there could be applications where there are great benefits of eliminating the need of fibre cabling at places where optical fibre is difficult to install [6]. As shown in Fig. 1, this solution effectively allows a centralised DDAS architecture for wireless distribution and coverage extension through $\mathrm{mmW}$ wireless channels in order to enable fast rollout of neutral-host CRANs with low installation costs. However, for such a system to be fully cost effective overall, multiple service operation, rather than single service operation is required.

In this paper therefore, we experimentally demonstrate simultaneous transmission of two 64 quadrature amplitude modulation (QAM) LTE services over a 1.25 Gbps digital RoF link to highlight the capabilities of carrying multiple digitised wireless services on a single infrastructure in a spectral and cost efficient way. It is shown that the $\mathrm{mmW}$ carrier can potentially accommodate several compressed 
DRoF bands or services operating on a single carrier. Thus, we show a multi-service digital signal over $\mathrm{mmW}$ architecture allowing several $2 / 3 / 4 G$ services to be delivered wirelessly without compromising radio frequency $(\mathrm{RF})$ performance.

\section{THEORY}

\section{A. System Architecture}

As shown in Fig. 2, the system architecture is composed of a DRoF access unit (AU), where wireless services as well as BBU CPRI signals are combined and processed together before optical transmission, a pair of $\mathrm{mmW}$ transceivers at either end of the wireless link performing up/down conversions and creating a wireless relay channel in the $\mathrm{mmW}$ band, and a DRoF remote unit (RU) providing RF coverage. In the downlink, RF signals are firstly down converted to an intermediate frequency (IF) before digitisation and data compression in the AU. The compressed low bit-rate data carrying the RF services are then up converted to the $\mathrm{mmW}$ band at the $\mathrm{mmW}$ transmitter to create a point-to-point wireless relay link. After wireless transmission, the data is subsequently recovered, and then retransmitted for RF distribution.

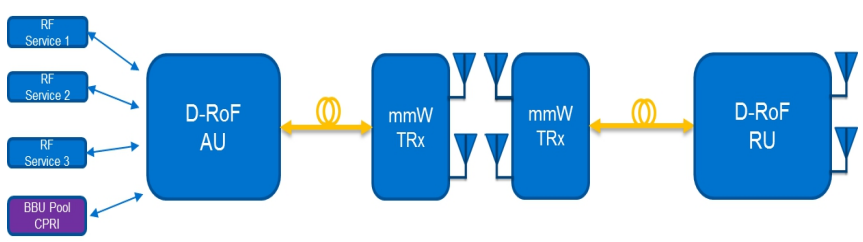

Fig. 2. Archietcutre for the digital RoF over mmW testbed

Transmission of data using millimetre waves with carrier frequencies ranging from $30-300 \mathrm{GHz}$ is a promising approach to overcome known data traffic demands, having greater capacity problems in the low frequency bands [7-8]. Currently, there is particular interest in using the $\mathrm{W}$-band $(75-110 \mathrm{GHz})$ where large bandwidths and licence free spectrum are available.

\section{B. Bandwidth Considerations}

This paper focuses on LTE signals to demonstrate the capability of carrying several wideband carriers. Commercially available $10 \mathrm{Gbps} \mathrm{mmW}$ links at $70 \mathrm{GHz}-$ $80 \mathrm{GHz}$ have been made available through ELVA-1 and Cablefree with 128/256 quadrature amplitude modulation (QAM) [9-10]. However, their spectral efficiency is limited by CPRI for RF fronthaul transmission. The data compression technique developed in [5] requires a 400 Mbps data rate per $20 \mathrm{MHz}$ LTE. In this case, a $10 \mathrm{Gbps}$ link can accommodate 25 LTE channels (including MIMO channels). By comparison, the current CPRI standard is not able to support that number of LTE channels as 25 LTE bands require at least 31.25 Gbps throughput which is beyond the highest data rate specified [3]. The number of services supported by a 10 Gbps link will be higher if lower bandwidth services such as WCDMA (5 MHz) and GSM $(200 \mathrm{kHz})$ are to be provided.

\section{EXPERIMENTAL SETUP}

The experimental setup consists of a DRoF testbed and a $\mathrm{mmW}$ up/down conversion testbed. In the DRoF testbed shown in Fig 3, two $20 \mathrm{MHz} 64 \mathrm{QAM}, 15.36 \mathrm{Msymbol} / \mathrm{s}$ LTE compatible signals at a $37.5 \mathrm{MHz}$ IF frequency are generated by Rhode and Schwarz (R\&S) SMBV100A vector signal generators (VSG). In practice, the RF services located in different frequency bands would first be down converted to the same IF frequency by an individual RF frontend before digitisation. We use the SMBV100A VSG to replace the RF frontend in the experimental setup. The IF signals are subsequently digitised by a pair of 14bit $150 \mathrm{Msample/s}$ analogue-to-digital converters (ADI AD9254 ADCs) which produce digital bits at 2.1 Gbps rates for each channel. The ADC cards are connected to an

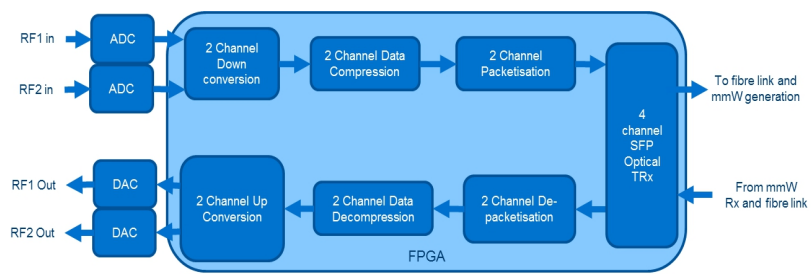

Fig. 3. Block diagram for the digitised radio signal experimental setup

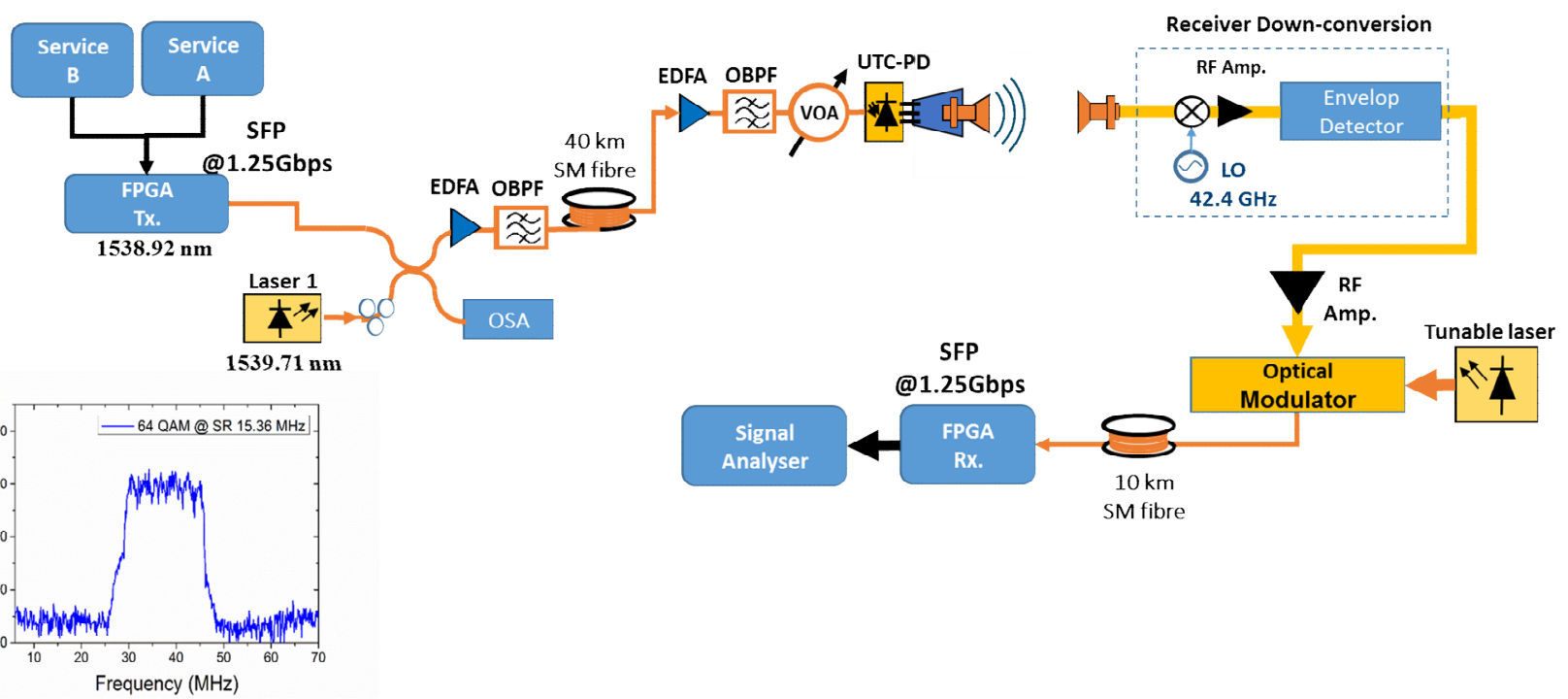

Fig.4. Optical $\mathrm{mmW}$ generation and detection testbed for wireless bridge experiment. 
Altera Stratix IV field programmable gate array (FPGA) development board through a high-speed mezzanine card (HSMC) interface via a low voltage differential signalling (LVDS) module. On the FPGA, the digitised IF data is firstly down converted to baseband in-phase and quadrature (IQ) information before data compression and packetisation take place. The compressed digitised IQ data is used to intensity modulate a $1538.95 \mathrm{~nm}$ distributed feedback (DFB) laser packaged in a small form factor plus $(\mathrm{SFP}+)$ optical transceiver. The aggregated data rate is now $800 \mathrm{Mbps}$ and a $1.25 \mathrm{Gbps}$ transceiver link is used to transport the information over optical fibre. In the reverse link, the received digital bits are de-packetised, decompressed and upconverted back to a digital IF format. A pair of 14-bit digital-to-analogue converters (Texas Instrument DAC5672 DACs) are used to recover the signal back to the analogue domain and the signal performance is tested by the R\&S FSW vector signal analyser (VSA). A utilisation report generated from the Altera's Quartus II software is shown on TABLE I. As seen, the total utilisation is below $1 / 3$ of the total FPGA capacity. In this case, at least 6 LTE channels can be supported on this single FPGA. For more services in the future, we can either select large volume FPGAs or multiple smaller ones running in parallel depending on the overall cost.

TABLE I. FPGA UTILISATION

\begin{tabular}{|l|c|}
\hline Logic utilization & $32 \%$ \\
\hline $\begin{array}{l}\text { Combinational adaptive lookup } \\
\text { tables (ALUTs) }\end{array}$ & $37,862 / 182,400$ \\
$(21 \%)$
\end{tabular}

In the up/down conversion $\mathrm{mmW}$ testbed shown in Fig. 4, the data stream is then coupled with an external cavity laser (ECL) at $1539.71 \mathrm{~nm}$, offset by $94 \mathrm{GHz}$ from the SPF+ output signal. Then, the optical output carrying the digital RF signals is amplified by an Erbium doped fibre amplifier (EDFA) and an optical bandpass filter (OBF) is used to remove the amplified spontaneous emission. The optical signal is then transmitted over $40 \mathrm{~km}$ single mode fibre (SMF) with $8 \mathrm{~dB}$ loss. To test the impact of the optical power level on the $\mathrm{mmW}$ performance, a variable optical amplifier (VOA) is employed to adjust the optical power before $\mathrm{mmW}$ generation by a uni-travelling carrier photodiode (UTC-PD). The UTC-PD is an unpackaged device with coplanar waveguide (CPW) contacts to convey the $\mathrm{mmW}$ signal onto one of a pair of directional horn antennas with $20 \mathrm{dBi}$ gain for wireless transmission. The antennas are separated by $1 \mathrm{~m}$, limited by the lab dimensions. In practice, the wireless transmission distance can be further extended until error free transmission is lost. At the receiver, the received $\mathrm{mmW}$ carrier is then down-converted by a sub-harmonic mixer (SHM) with an electrical local oscillator (LO) operating at 42.4 GHz. An envelope detector is used to remove the carrier frequency fluctuations and recover the baseband information by performing square law detection. The output is amplified and fed onto an optical modulator for electrical to optical conversion. Before returning to the FPGA for digital signal processing, the signal is transmitted over $10 \mathrm{~km}$ fibre link to demonstrate its capability of long range optical transmission without performance loss.

\section{EXPERIMENTAL RESULTS}

The error vector magnitude (EVM) measures the modulation accuracy of wireless signals. The $3^{\text {rd }}$ generation partnership project (3GPP) LTE standard has specified a maximum EVM value of $8 \%$ for $64 \mathrm{QAM}$, $12.5 \%$ for $16 \mathrm{QAM}$, and $17.5 \%$ for quadrature phase shift keying (QPSK), respectively [11]. Fig. 5 shows the EVM results against various input optical powers by varying the VOA and measuring the received photocurrent squared in the UTC-PD. The EVM for both services is maintained below $6 \%$ for photocurrent above $0.77 \mathrm{~mA}$ after which it starts to increase rapidly until the signals are undetectable. This shows that, at the maximum photocurrent tested, it should be possible to extend the $\mathrm{mmW}$ wireless link length to $2.9 \mathrm{~m}$, corresponding to $9.5 \mathrm{~dB}$ free space loss ( $20 \log \left(\mathrm{d}_{2} / \mathrm{d}_{1}\right)=9.5 \mathrm{~dB}$, where $\mathrm{d}_{1}$ here refers to $1 \mathrm{~m}$, and $\mathrm{d}_{2}$ is the new distance calculated for $9.5 \mathrm{~dB}$ extra free space losses in the link ).

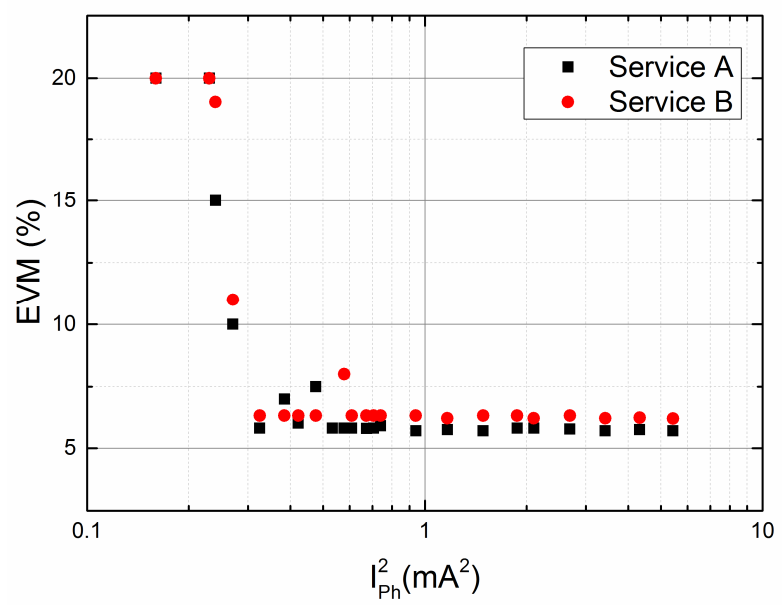

Fig. 5. EVM measurements versus photocurrent squared.

The RF performance is tested by measuring the dynamic range of signal input power when the EVM is below the requirement level. As shown in Fig. 6, the total dynamic range is around $40-50 \mathrm{~dB}$ for service $\mathrm{A}$, and 35-

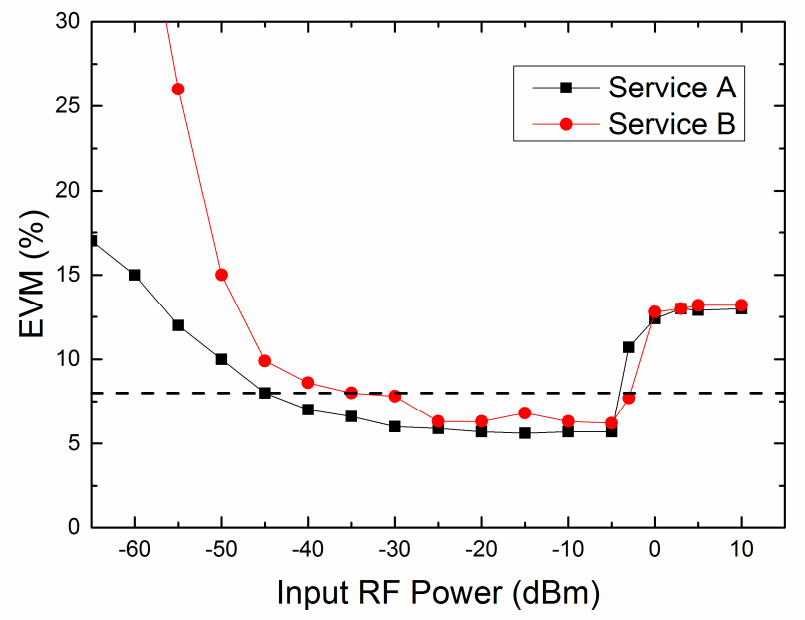

Fig. 6. EVM measurments versus RF input power at the DAC. 
$40 \mathrm{~dB}$ for service B for 64QAM LTE signals. The dynamic range difference between the two channels is due to a synchronisation code added to service B for data clock recovery at the FPGA. This code can lead to some errors in one of the two channels as a result of the internal synchronisation processing. A new synchronisation scheme is under investigation to completely remove the errors. Ideally, the two services will have the same dynamic range result. However, this will not impact the overall system dynamic performance when automatic gain control (AGC) is used at the RF frontend to compensate the dynamic range loss. It is expected over $65 \mathrm{~dB}$ dynamic range can be achieve with $30 \mathrm{~dB}$ AGC.

The received optical power at the $\mathrm{SFP}+$ receiver is measured to test the optical dynamic range in the system while keeping the RF input power for both services at -10 $\mathrm{dBm}$. As shown in Fig. 7, the EVM remains below 6\% between $0 \mathrm{dBm}$ and $-25 \mathrm{dBm}$, giving $25 \mathrm{~dB}$ optical dynamic range. When the received optical signal is higher than $0 \mathrm{dBm}$, the $\mathrm{SFP}+$ optical receiver is overloaded, and causes signal distortion. At the low received optical power below $-25 \mathrm{dBm}$, the DAC losses the synchronization causing error on the detectable data stream.

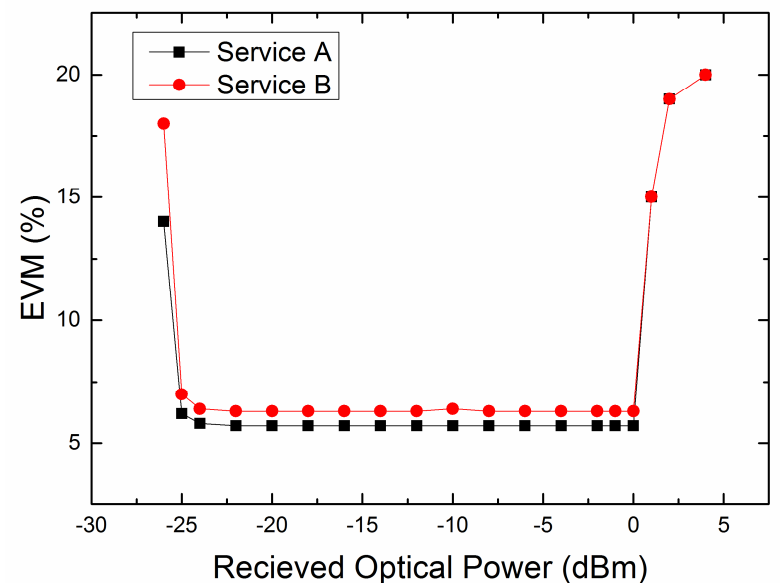

Fig. 7. EVM measurements versus received optical power at the SFP+'s optical receiver.

\section{CONCLUSiOnS}

This paper demonstrates a multichannel digital radio distribution system that utilises both optical fibre and wideband $\mathrm{mmW}$ infrastructure for wireless coverage. Despite being flexible in terms of transmission link, the system is able to efficiently accommodate multiple wireless carriers or services in a neutral host manner by performing digital data compression which shows 3-times higher efficiency than CPRI.

In the experimental setup, two 64-QAM LTE services are processed and transmitted over 1.25 Gbps digital links including $50 \mathrm{~km}$ of optical fibre and $1 \mathrm{~m}$ of free space using mmW carrier. The results show over $35 \mathrm{~dB}$ RF dynamic range and $25 \mathrm{~dB}$ optical dynamic range for both channels. The solution sustains high spectral efficiency as well as benefiting from the flexibility and costeffectiveness that digital systems provide in allowing a wide range of potential transmission media, both free space and wired. The solution is finally particularly attractive given the emergence of massive multiple input and multiple output (MIMO) and carrier aggregation (CA) techniques in standards such as LTE-A and beyond which will require several channels to be transmitted simultaneously.

\section{ACKNOWLEDGMENT}

This work was funded by the UK Engineering and Physical Sciences Research Council grants for Converged Optical \& Wireless Access Networks (COALESCE) EP/P003990/1, and Coherent Terahertz Systems (COTS), $\mathrm{EP} / \mathrm{J} 017671 / 1$.

\section{REFERENCES}

[1] M.J. Crisp, Sheng Li, A. Watt, R.V. Penty, I.H. White, "Uplink and Downlink Coverage Improvement of 802.11g Signals Using a Distributed Antenna Network", Journal of Lightwave Technology, Vol.25, Issue 11, Nov. 2007 p3388-3395.

[2] T. Li, M. Crisp, R.V. Penty, I.H. White, "Low Bit Rate Digital Radio over Fibre System," Microwave Photonics, 2009. MWP '09.International Topical Meeting on , vol., no., pp.1,4, 14-16 Oct. 2009

[3] CPRI Specification, V 7.0, Oct 2015.

[4] eCPRI Specification, V1.1 Jan 2018.

[5] T. Li R.V. Penty I.H. White "Novel digital radio over fibre for 4GLTE" IEEE Int. Conf. Communication Workshop (ICCW) pp. 312317 June 2015

[6] H. Shams, T. Li, C. C. Renaud, A. J. Seeds, R. Penty, M. Fice, and I. White, "Digital Radio over Fiber Distribution using Millimetre Wave Bridging," in Optical Fiber Communication Conference, 2018.

[7] C. Dehos, J. L. González, A. De Domenico, D. Kténas, and L. Dussopt, "Millimeter-Wave Access and Backhauling : The Solution to the Exponential Data Traffic Increase in 5G Mobile Communications Systems ?," no. September, pp. 88-95, 2014.

[8] P. T. Dat, A. Kanno, and T. Kawanishi, "Radio-on-radio-overfiber: Efficient fronthauling for small cells and moving cells," IEEE Wirel. Commun., vol. 22, no. 5, pp. 67-75, 2015.

[9] ELVA-1, "10 Gigabit Ethernet MM-wave Link", PPC-10g series datashsheet, March 2017.

[10] Cablefree, "Millimeter-wave radio (MMW) MMW Radio 70/80GHz, 10Gbps Ethernet", Wireless Exellence datasheet, 2016.

[11] 3GPP TS 36.101 V12.6.0, Evolved Universal Terrestrial Radio Access (E-UTRA) User Equipment (UE) Radio Transmission and Reception (Release 12), December 2014 